\title{
Remarques sur le spectre de l'opérateur de Dirac
}

\author{
Nicolas Ginoux
}

21 janvier 2021

Résumé : Nous décrivons un nouvelle famille d'exemples d'hypersurfaces de la sphère satisfaisant le cas d'égalité de la majoration extrinsèque de C. Bär de la plus petite valeur propre de l'opérateur de Dirac.

\section{Remarks on the spectrum of the Dirac operator}

\begin{abstract}
We describe a new family of examples of hypersurfaces in the sphere satisfying the limitingcase in C. Bär's extrinsic upper bound for the smallest eigenvalue of the Dirac operator.
\end{abstract}

Soit $\left(M^{m}, g\right)$ une hypersurface riemannienne compacte orientée (de dimension $m$ ) de l'espace-modèle $\left(\widetilde{M}^{m+1}(c), g\right)$, où $\widetilde{M}^{m+1}(0):=\mathbb{R}^{m+1}$ (l'espace euclidien), $\widetilde{M}^{m+1}(1):=S^{m+1}$ (la sphère ronde à courbure sectionnelle 1 ), et $\widetilde{M}^{m+1}(-1):=\mathbb{H}^{m+1}$ (l'espace hyperbolique à courbure sectionnelle -1 ). Soit $\lambda_{1}$ la plus petite valeur propre de l'opérateur de Dirac sur $M$ associé à $g$ et à la structure spinorielle induite. Il est en général impossible de donner une expression explicite pour cette valeur propre. Il a cependant été démontré que la courbure moyenne $H$ ainsi que la courbure ambiante la majorent de manière naturelle; plus précisément, C. Bär a montré dans [2] que

$$
\begin{array}{ll}
\lambda_{1}^{2} \leq \frac{m^{2}}{4 \operatorname{Vol}(M)} \int_{M} H^{2} v_{g} & \text { si } \widetilde{M}=\mathbb{R}^{m+1}, \\
\lambda_{1}^{2} \leq \frac{m^{2}}{4 \operatorname{Vol}(M)} \int_{M}\left(H^{2}+1\right) v_{g} & \text { si } \widetilde{M}=S^{m+1},
\end{array}
$$

et l'auteur a prouvé dans $[11,10]$ que

$$
\lambda_{1}^{2} \leq \frac{m^{2}}{4}\left(\sup _{M} H^{2}-1\right) \quad \text { si } \widetilde{M}=\mathbb{H}^{m+1} .
$$

Si (1) et (2) (resp. (3)) montrent une forte analogie avec les majorations de R.C. Reilly [16] (resp. de E. Heintze [12]) pour la première valeur propre non nulle du laplacien scalaire, on peut se demander jusqu'où va cette ressemblance, et si en particulier l'égalité dans (1), (2) ou (3) n'est atteinte que lorsque $M$ est minimalement immergée dans une sphère géodésique, comme c'est le cas pour le laplacien $[16,12,6]$. Il a été démontré dans [2] (resp. dans [11]) que, si (1) ou (2) (resp. (3)) est une égalité, alors la courbure moyenne $H$ est constante, et que l'égalité a lieu si $M$ est une sphère géodésique; néanmoins, le problème de savoir si cette hypersurface est la seule à jouir de cette propriété est à ce jour demeuré ouvert.

Nous montrons dans cette note que l'égalité dans (2) lorsque $M$ n'est pas une sphère géodésique n'entraine pas la minimalité de $M$ dans $S^{m+1}$. Nous exhibons une famille d'exemples à cet effet.

Ce travail a été effectué à l'Institut Max-Planck pour les Mathématiques dans les Sciences de Leipzig, que l'auteur tient à remercier pour son soutien et son hospitalité. C'est aussi un plaisir de remercier Oussama Hijazi pour son soutien et pour sa lecture critique de l'article.

\section{Structures spinorielles sur les sous-variétés}

Pour les préliminaires sur la géométrie spinorielle, on se reportera par exemple à [13, 5, 4, 8]. 
Nous commençons par rappeler quelques éléments de base sur la restriction de structures spinorielles (cf. aussi $[2,15,10])$. Tout d'abord, si $E$ et $F$ sont deux fibrés vectoriels riemanniens sur une variété donnée, la présence d'une structure spinorielle sur deux des fibrés $E, F, E \oplus F$, induit une structure spinorielle sur le troisième ([14] et [13], Prop. 2.15 p. 90). Cela a deux conséquences importantes dans les exemples que nous allons étudier.

D'une part, tout produit riemannien de deux variétés spinorielles porte une structure spinorielle naturelle appelée structure spinorielle produit. On peut même montrer que toute structure spinorielle sur un produit riemannien est en fait une structure spinorielle produit.

D'autre part, toute sous-variété riemannienne à fibré normal trivial d'une variété riemannienne spinorielle porte une structure spinorielle induite. Nous insistons ici sur le fait que cette structure spinorielle dépend de la trivialisation du fibré normal choisie [3]. C'est pourquoi nous préciserons toujours ce choix.

Nous aurons également besoin de l'observation suivante. Soient $E_{1} \longrightarrow M^{\prime}$ et $E_{2} \longrightarrow M^{\prime}$ deux fibrés vectoriels réels triviaux sur une variété $M^{\prime}$, de rangs respectifs $n_{1}$ et $n_{2}$. Soit $\left(X_{1}, \ldots, X_{n_{1}}\right)$ (resp. $\left.\left(X_{n_{1}+1}, \ldots, X_{n_{1}+n_{2}}\right)\right)$ une trivialisation de $E_{1}$ (resp. de $E_{2}$ ) et supposons la variété $M^{\prime}$ spinorielle. Toute trivialisation d'un fibré vectoriel munissant celui-ci de la structure spinorielle triviale, $\left(X_{1}, \ldots, X_{n_{1}}\right)$ (resp. $\left.\left(X_{n_{1}+1}, \ldots, X_{n_{1}+n_{2}}\right)\right)$ induit d'après ce qui précède une structure spinorielle sur le fibré $T M^{\prime} \oplus E_{1}$ (resp. sur $\left.T M^{\prime} \oplus E_{2}\right)$. De même, la trivialisation $\left(X_{1}, \ldots, X_{n_{1}+n_{2}}\right)$ de $E_{1} \oplus E_{2}$ induit une structure spinorielle sur $T M^{\prime} \oplus\left(E_{1} \oplus E_{2}\right)$. Mais $T M^{\prime} \oplus\left(E_{1} \oplus E_{2}\right)=\left(T M^{\prime} \oplus E_{1}\right) \oplus E_{2}$, donc $T M^{\prime} \oplus\left(E_{1} \oplus E_{2}\right)$ porte aussi la structure spinorielle induite par celle de $T M^{\prime} \oplus E_{1}$ et $\operatorname{par}\left(X_{n_{1}+1}, \ldots, X_{n_{1}+n_{2}}\right)$. La remarque est que ces deux structures coïncident. Cela s'applique en particulier à la restriction de structures spinorielles à une sous-variété à fibré normal trivial (voir ci-dessous).

\section{Une famille d'exemples d'hypersurfaces non minimales satis- faisant l'égalité dans (2)}

Nous décrivons maintenant les exemples cités en introduction. Soient $p, q$ deux entiers naturels non nuls, et $r, r^{\prime}$ deux réels strictement positifs. Considérons la variété $M:=S^{p}(r) \times S^{q}\left(r^{\prime}\right)$, où $S^{k}(R)$ désigne la sphère ronde de dimension $k$ et de rayon $R$. Notons $g$ la métrique produit sur $M$, et munissons $M$ de la structure spinorielle produit. Rappelons que, lorsque $k>1$, il n'existe qu'une seule structure spinorielle sur $S^{k}(R)$, alors que si $k=1$, la variété $S^{k}(R)$ porte deux structures spinorielles non équivalentes, une triviale et une non triviale. Cette dernière est celle qui est induite par le plongement standard $S^{1}(R) \subset \mathbb{R}^{2}$. Nous choisirons toujours lorsque $k=1$ la structure spinorielle non triviale sur $S^{k}(R)$.

Pour la structure spinorielle produit que l'on a fixée sur $M$, le spectre du carré de l'opérateur de Dirac $D_{M}$ de $(M, g)$ est l'ensemble des $\mu_{k}^{2}+\mu_{l}^{\prime 2}$, où $\mu_{k}$ (resp. $\left.\mu_{l}^{\prime}\right)$ décrit le spectre de l'opérateur de Dirac sur $S^{p}(r)$ (resp. sur $S^{q}\left(r^{\prime}\right)$ ) (cela est une propriété générale des produits riemanniens, cf. [1]). En utilisant [17], le spectre de $D_{M}^{2}$ est donc

$$
\left\{\frac{1}{r^{2}}\left(\frac{p}{2}+n\right)^{2}+\frac{1}{r^{\prime 2}}\left(\frac{q}{2}+n^{\prime}\right)^{2}, \quad n, n^{\prime} \in \mathbb{N}\right\}
$$

En particulier, la plus petite valeur propre de $D_{M}^{2}$ est $\lambda_{1}^{2}=\frac{p^{2}}{4 r^{2}}+\frac{q^{2}}{4 r^{\prime 2}}$.

Pour un réel $u$ dans $] 0, \frac{\pi}{2}\left[\right.$, posons $r:=\cos (u), r^{\prime}:=\sin (u)$, et renotons $M_{u}:=S^{p}(\cos (u)) \times S^{q}(\sin (u))$. La variété $\left(M_{u}, g\right)$ se plonge alors canoniquement et isométriquement dans $S^{p+q+1}(1)$. De plus, la structure spinorielle induite par ce plongement coïncide avec la structure spinorielle produit que l'on a fixée sur $M_{u}$. Pour montrer cela, distinguons deux cas.

a) Si $p>1$ et $q>1$, la structure spinorielle de $M_{u}$ est unique car $M_{u}$ est simplement connexe.

b) Si $p=1$ (le raisonnement sera identique si $q=1$ ), la situation est un peu plus compliquée puisque $M_{u}$ porte plusieurs structures spinorielles. Considérons le diagramme commutatif suivant dans lequel les flèches sont les inclusions standard : 


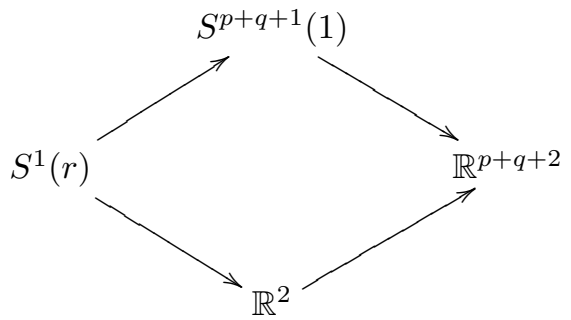

Nous devons déterminer si la structure spinorielle induite par $S^{p+q+1}(1) \operatorname{sur} M_{u}$ se restreint à son tour sur $S^{1}(r)$ en une structure spinorielle triviale ou non, cette dernière restriction étant réalisée au moyen de la trivialisation naturelle (compatible avec les orientations) du fibré normal de $S^{1}(r)$ dans $M_{u}$. Par définition, la structure spinorielle induite par $S^{p+q+1}(1)$ sur $M_{u}$ est donnée par la trivialisation du fibré normal de $M_{u}$ dans $S^{p+q+1}(1)$; par suite (cf. paragraphe 1), celle induite par $M_{u}$ sur $S^{1}(r)$ est également induite par $S^{p+q+1}(1)$ sur $S^{1}(r)$ au moyen de la trivialisation de son fibré normal dans $S^{p+q+1}(1)$. Or la structure spinorielle de $S^{p+q+1}(1)$ provient elle-même de la structure spinorielle de $\mathbb{R}^{p+q+2}$ par l'inclusion canonique $S^{p+q+1}(1) \subset \mathbb{R}^{p+q+2}$; la structure spinorielle induite par $M_{u}$ sur $S^{1}(r)$ est donc aussi celle induite sur $S^{1}(r)$ par l'inclusion canonique $S^{1}(r) \subset \mathbb{R}^{p+q+2}$ au moyen de la trivialisation naturelle du fibré normal de ce plongement.

Mais cette dernière trivialisation est également obtenue en juxtaposant les trivialisations des fibrés normaux respectifs de $S^{1}(r)$ dans $\mathbb{R}^{2}$ et de $\mathbb{R}^{2}$ dans $\mathbb{R}^{p+q+2}$ (où $\mathbb{R}^{2}=\mathbb{R}^{2} \times\{0\} \subset \mathbb{R}^{p+q+2}$ ); comme $\mathbb{R}^{2}$ porte évidemment la structure spinorielle induite par la trivialisation canonique de son fibré normal dans $\mathbb{R}^{p+q+2}$, et que le plongement naturel $S^{1}(r) \subset \mathbb{R}^{2}$ induit une structure spinorielle non triviale sur $S^{1}(r)$, la structure spinorielle induite sur $M_{u}$ induit donc à son tour une structure spinorielle non triviale sur chacun des facteurs de dimension 1.

Par conséquent, la première valeur propre $\lambda_{1}$ de l'opérateur de Dirac de $M_{u}$ pour la structure spinorielle induite satisfait

$$
\lambda_{1}^{2}=\frac{p^{2}}{4 \cos (u)^{2}}+\frac{q^{2}}{4 \sin (u)^{2}} .
$$

Or un calcul élémentaire du carré de la courbure moyenne de $M_{u}$ dans $S^{p+q+1}(1)$ donne

$$
H^{2}=\frac{1}{(p+q)^{2}}\left(p \tan (u)-\frac{q}{\tan (u)}\right)^{2},
$$

d'où

$$
\lambda_{1}^{2}=\frac{(p+q)^{2}}{4}\left(H^{2}+1\right),
$$

ce qui n'est rien d'autre que l'égalité dans (2).

Nous obtenons donc une situation radicalement différente de celle du laplacien scalaire, puisqu'il n'existe qu'un seul $u$ dans $] 0, \frac{\pi}{2}$ [ pour lequel la première valeur propre non nulle du laplacien scalaire de $\left(M_{u}, g\right)$ satisfait l'égalité dans la majoration de R.C. Reilly [16], à savoir celui pour lequel $M_{u}$ est minimale dans $S^{p+q+1}(1)$. Cette dissemblance, qui vient s'ajouter à celle déjà observée par l'auteur [11] sur la question d'une possible amélioration de (3) en une majoration $L^{2}$ optimale (cf. aussi [9]), semblerait indiquer une plus grande diversité de résultats pour l'opérateur de Dirac dans les problèmes d'estimations extrinsèques des petites valeurs propres. Ce dernier point rejoint par ailleurs la différence observée pour l'étude du cas d'égalité dans la minoration (intrinsèque) de $\mathrm{T}$. Friedrich [7] de la plus petite valeur propre de l'opérateur de Dirac en fonction de la courbure scalaire (voir $[5,8]$ pour ces questions).

\section{Références}

[1] M.F. Atiyah, Eigenvalues of the Dirac operator, Lect. Notes in Math. 1111 (1985), 251-260.

[2] C. Bär, Extrinsic Bounds for Eigenvalues of the Dirac Operator, Ann. Glob. Anal. Geom. 16 (1998), 573-596.

[3] _ discussions privées.

[4] H. Baum, T. Friedrich, R. Grunewald, I. Kath, Twistor and Killing Spinors on Riemannian Manifolds, Teubner-Texte zur Mathematik 124 (1991), Teubner-Verlag Stuttgart/Leipzig. 
[5] J.-P. Bourguignon, O. Hijazi, J.-L. Milhorat, A. Moroianu, A Spinorial approach to Riemannian and Conformal Geometry (en préparation).

[6] A. El Soufi, S. Ilias, Une inégalité de type Reilly pour les sous-variétés de l'espace hyperbolique, Comment. Math. Helv. 67 n 22 (1992), 167-181.

[7] T. Friedrich, Der erste Eigenwert des Dirac-Operators einer kompakten riemannschen Mannigfaltigkeit nichtnegativer Skalarkrümmung, Math. Nach. 97 (1980), 117-146.

[8] _ Dirac operators in Riemannian geometry, Graduate Studies in Mathematics 25 (2000), American Mathematical Society.

[9] N. Ginoux, Reilly-type spinorial inequalities, Math. Zeit. 241 n 30 (2002), 513-525.

[10] _ Opérateurs de Dirac sur les sous-variétés, Thèse de doctorat (2002), Université Henri Poincaré, Nancy.

[11] _ Une nouvelle estimation extrinsèque du spectre de l'opérateur de Dirac (soumis).

[12] E. Heintze, Extrinsic upper bound for $\lambda_{1}$, Math. Ann. 280 (1988), 389-402.

[13] H.B. Lawson, M.-L. Michelsohn, Spin Geometry, Princeton University Press (1989).

[14] J. Milnor, Remarks concerning spin manifolds, Princeton University Press (1965), 55-62.

[15] B. Morel, Eigenvalue Estimates for the Dirac-Schrödinger Operators, J. Geom. Phys. 38 (2001), $1-18$.

[16] R.C. Reilly, On the first eigenvalue of the Laplacian for compact submanifolds of Euclidean space, Comment. Math. Helv. 52 (1977), 525-533.

[17] S. Sulanke, Berechnung des Spektrums des Quadrates des Dirac-Operators auf der Sphäre und Untersuchungen zum ersten Eigenwert von $D$ auf 5-dimensionalen Räumen konstanter positiver Schnittkrümmung, Dissertation (1981), Humboldt-Universität, Berlin.

Nicolas GINOUX

Universität Hamburg, FB Mathematik - SPAD, Bundesstraße 55 D-20146 Hamburg

fmcw191@math.uni-hamburg.de 